\title{
La trayectoria de cuidado del paciente en hemodiálisis: de la noticia inesperada al desenlace final
}

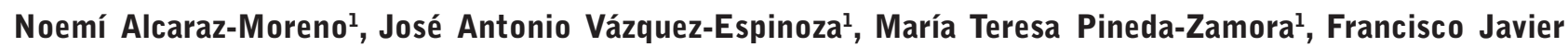 \\ Ramos-Sánchez ${ }^{2}$
}

'Facultad de Enfermería. Universidad de Colima. Colima. México

${ }^{2}$ Centro Universitario del Sur. Universidad de Guadalajara. México

Como citar este artículo:

Alcaraz-Moreno N, Vázquez-Espinoza JA, Pineda-Zamora MT, Ramos-Sánchez Francisco J. La trayectoria de cuidado del paciente en hemodiálisis: de la noticia inesperada al desenlace final.

Enferm Nefrol. 2019 Jul-Sep;22(3):308-16

Introducción: Las complicaciones de la enfermedad renal crónica y su tratamiento degeneran el estado de salud del paciente condicionando su dependencia en el autocuidado de su salud, lo que desencadena la necesidad de cuidado de otra persona, por lo que, desde el momento en que el paciente y su familia reciben el diagnóstico y la noticia del tratamiento, llevan a cabo un proceso que los llevará a afrontar la nueva situación que se les presenta.

Objetivos: Describir las vivencias de las cuidadoras de pacientes en hemodiálisis, para generar estrategias e intervenciones de enfermería derivadas de sus necesidades de apoyo.

Material y Método: Investigación cualitativa, mediante el empleo de la teoría fundamentada, la recolección de información fue mediante la entrevista en profundidad de tipo semiestructurada y la observación participante. Resultados: La trayectoria de cuidado que experimenta el cuidador primario del paciente en hemodiálisis atraviesa por tres etapas: el inicio, donde a través de la noticia inesperada se convierte en cuidador y experimenta una serie de cambios que envuelven su vida personal, familiar y social. Una etapa intermedia, en donde lleva a cabo un proceso de adaptación y da significado al

\footnotetext{
Correspondencia:

José Antonio Vázquez Espinoza

Facultad de Enfermería. Universidad de Colima

Av. Universidad 333, Col. Las víboras; c.p 28040 Colima. México. E-mail:TONYCEIN@hotmail.com
}

cuidado que brinda a su familiar y una etapa final, en donde enfrenta diferentes tipos de duelo.

Conclusión: El aceptar la situación, asumir su rol de cuidadoras, el tiempo y la creencia en un ser superior fueron los recursos de donde obtuvieron la fortaleza para afrontar los procesos por lo que pasaran en cada una de las etapas de la trayectoria de cuidado.

PALABRAS CLAVE: cuidador primario; enefermería; hemodiálisis.

\section{The trajectory of patient care in hemodialysis: From the unexpected news to the final outcome}

Introduction: Complications of chronic kidney disease and treatment degenerate the patient's state of health, conditioning independence in health self-care. Situation that triggers the need for care of another person. From the moment that the patient and the family receive the diagnosis and the news of the treatment, they carry out a process that will lead them to face the new situation that is presented.

Objectives: To describe the caregivers' experience in hemodialysis patients, to generate strategies and nursing interventions derived from their support needs.

Material and Method: Qualitative research, using grounded theory, the collection of information was carried out through an in-depth semi-structured interview and participant observation. 
Results: The care path experienced by the primary caregiver of the hemodialysis patient goes through three stages: the first stage, after the unexpected news becomes a caregiver and experiences a series of changes that involve the personal, family and social sphere. An intermediate stage, in which there is a process of adaptation and gives meaning to the care provided to your family member; and a final stage, where different types of grief are faced.

Conclusions: Accepting the situation, assuming the role of caregivers, time and belief in a superior being were the resources from which they obtained the got the strength to face the processes that they will go through in each of the stages of the care path.

KEYWORDS: primary caregiver; nurse; hemodialysis.

\section{Introducción}

La Enfermedad Renal Crónica (ERC) representa un problema de salud que según el estudio de epidemiología de la IRC en México afecta al menos 40,000 casos nuevos cada año, además de que ocupa el décimo lugar como causa de muerte, contribuyendo con el $2 \%$ $(13,167)$ de todas las muertes de nuestro país ${ }^{1}$.

El aumento de casos de ERC ha condicionado el uso de las terapias de sustitución renal como la hemodiálisis. Las complicaciones de la ERC y su tratamiento degeneran el estado de salud del paciente generando dependencia en el cuidado de su salud, lo que desencadena la necesidad de cuidado de otra persona ${ }^{2}$. Los familiares adoptan el rol de cuidador primario, siendo generalmente mujeres quienes asumen dicho rol principalmente, las cuales tienen como parentesco hijas, esposas y madres del paciente, tienen educación básica incompleta, sin trabajo, sin participación social y con percepción económica insuficiente 3 .

Desde el momento en que el paciente y su familia reciben el diagnóstico y la noticia de que el tratamiento consiste en la hemodiálisis, ambos llevan a cabo un proceso que los llevará a afrontar la nueva situación que se les presenta.

Diversos estudios han demostrado los cambios y repercusiones que el cuidado informal ocasiona en la vida de los cuidadores, convergen en que el rol de cuidador primario es sumamente difícil, ya que tienen que asumir una gran cantidad de tareas que los pone en una situación de gran vulnerabilidad, estrés y carga y que incrementa, a su vez, el riesgo de padecer problemas físicos, emocionales, sociales, familiares y otros. Las repercusiones del cuidado influyen en la calidad de vida del cuidador ${ }^{4-5}$.

Es, en ese sentido, que nuestro estudio considera como punto de partida al cuidador primario, quien es quien vive la experiencia de cuidar. Por lo tanto, es imperante que los profesionales de la salud tomen medidas enfocadas en apoyo a los cuidadores. Asimismo, consideramos que los resultados obtenidos podrán ser empleados para generar estrategias e intervenciones de enfermería derivadas de sus necesidades de apoyo. Pues al comprender sus experiencias, los profesionales de enfermería podrán visualizar las necesidades propias de las cuidadoras, y de esa manera potenciar sus habilidades para el cuidado, lo que sin duda coadyuvara a disminuir los efectos adversos del cuidado en su vida.

Aun cuando se ha descrito las consecuencias que experimentan como cuidadores primarios se desconoce cómo viven la experiencia de cuidar a su familiar enfermo cuando son sometidos al tratamiento de hemodiálisis, por lo que este estudio tuvo como objetivo describir las vivencias de las cuidadoras de pacientes en hemodiálisis.

\section{Material y Método}

Se realizó una investigación cualitativa, mediante el empleo de la teoría fundamentada. Participaron familiares de pacientes en tratamiento de hemodiálisis que desempeñaban el rol de cuidador primario por más de tres meses, mayores de edad, y que previo consentimiento informado aceptaron participar en la investigación y otorgaron su firma.

La investigación fue realizada en dos escenarios. El Centro Estatal de Hemodiálisis del estado de Colima, México, donde se autorizó el desarrollo de la investigación, y se obtuvo la lista de pacientes en hemodiálisis; a través del listado estableció contacto con sus cuidadores. El otro escenario fueron los hogares de los cuidadores; se agendaron citas vía telefónica y se les hizo una visita para invitarlos a participar en esta investigación, ahí mismo se realizaron las entrevistas.

La recolección de la información fue mediante la entrevista en profundidad de tipo semiestructurada. Además, se realizaron registros en cada una de las entrevistas de los sentimientos y emociones observadas en las participantes. Las entrevistas tuvieron un tiempo 
de duración promedio de una hora y fueron grabadas para su posterior análisis. En la recolección de las vivencias de las cuidadoras se empleó la saturación teórica, por lo que en algunos cuidadores se

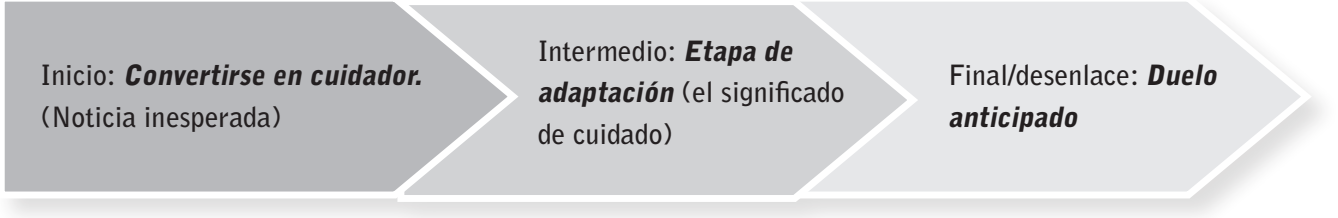

Figura 1. Trayectoria del cuidado de pacientes en hemodiálisis: Voz de sus cuidadores primarios. realizaron hasta tres entre-

vistas, las cuales se llevaron a cabo en dos momentos. En el primero se recabó información respecto a las vivencias, realizándose su respectivo análisis, lo que nos dio la pauta a indagar en las cuidadoras la etapa final de la trayectoria del cuidado. Cabe señalar que la primera recolección se llevó a cabo en el segundo semestre del año 2016 y la segunda en el 2017.

Las informantes del estudio fueron 14 cuidadores de pacientes en tratamiento de hemodiálisis, 13 mujeres y un hombre, con edades entre 20 y 70 años, que se dedicaban al hogar. Las cuidadoras tienen parentesco de hijas, madres y esposas de los pacientes. Dichos pacientes tienen un tiempo de 4 meses hasta 2 años con 10 meses en el tratamiento de hemodiálisis, y los cuidadores tienen de 2 a 7 años siendo cuidadores primarios.

El análisis de la información se realizó mediante la propuesta de la Teoría Fundamentada. El análisis se inició después de cada entrevista, se transcribieron y posteriormente se inició con una lectura general a la entrevista transcrita, para tener una idea general sobre la misma, seguido de lectura de párrafo por párrafo para la comprensión y la identificación de datos significativos. Posterior a la identificación de datos significativos elaboramos fichas bibliográficas donde codificamos y realizamos memos analíticos y de trabajo. La realización de memos analíticos apoyaron las comparaciones con la teoría y los de trabajo se usaron para identificar las tareas que surgían del análisis. Posterior al análisis y teniendo un total de 350 códigos aproximadamente, buscamos la relación entre cada uno de ellos y elaboramos 12 familias, de ellas emergieron las primeras categorías, a partir de las cuales se desarrollaron esquemas en busca de asociaciones entre ellas y finalmente las refinamos. Cabe señalar que en todo este proceso empleamos el método de comparación constante, es decir, tomar a la literatura como una fuente de datos.

Se han respetado en cada una de las etapas de la investigación los preceptos éticos para la investigación en seres humanos, tales como el consentimiento informado, la confidencialidad de los participantes y el manejo de las grabaciones ${ }^{6-8}$.

\section{Resultados y Discusión}

Al centrar la mirada en los cuidadores primarios, se ha identificado que ha transcurrido un camino desconocido para ellos. En un inicio dicho camino lo fueron descubriendo con las experiencias del diario vivir y con el cuidado y acompañamiento de sus familiares en tratamiento en hemodiálisis. Asimismo, han caminado por un sendero donde se presentó la adaptación a los cambios implicados en el cuidado de su familiar, para finalmente experimentar una etapa de duelo anticipado ante la presente idea de la muerte de su familiar. La Figura 1 muestra el proceso que hemos denominado trayectoria del cuidado.

\section{Categoría 1.- Convertirse en Cuidador}

De esta manera comienza la trayectoria del cuidado que vivieron los cuidadores primarios de pacientes en hemodiálisis:

"iAy fue terrible!, porque pues no nos lo esperábamos, yo me puse a llorar..." E_3

"Ay pues fue bien difícil. Fue difícil porque pues no nos esperábamos pues la enfermedad ésta" E_3

Los cuidadores recibieron la inesperada noticia sobre el tratamiento en hemodiálisis de sus familiares. En esos momentos experimentaron un cúmulo de emociones, de incertidumbre, angustia y sorpresa.

Ante dicha noticia y las emociones que surgieron, los cuidadores vivieron la aceptación de que su familiar tenía una enfermedad crónica, la cual requiere de un tratamiento permanente como la hemodiálisis.

"No me quedaba de otra más que le hicieran eso" E_6

Los cuidadores tuvieron pensamientos que determinaron su manera de afrontar la situación. Pérez et al., refieren que ante esa situación se pueden tener pensamientos de vulnerabilidad, confusión y preocupación por la salud y el futuro de los familiares ${ }^{9}$. Los cuidadores de este estudio eligieron apropiarse de la condición de salud de su familiar y asumieron el rol de cuidadores 
luego de aceptar la nueva situación, con lo que se originó así a su proceso de afrontamiento:

"Tuve que yo aceptarlo en primer lugar, era lo que nos tocaba vivir [...] Yo estoy consciente de ello, yo al principio no aceptaba, pero lo he aceptado así, para que duela menos" $E_{-}$.

Expresiones como las de esta cuidadora dan muestra que el reconocer el inicio de un buen proceso de afrontamiento es aceptar la situación, lo que podría desencadenar en una vivencia menos dolorosa. Dicho afrontamiento se conoce como afrontamiento activo, y se describe como todas aquellas actividades que realiza el cuidador con la finalidad de resolver la situación o problema que se presente ${ }^{10}$.

Los cuidadores pasaron por momentos de dificultad, sin embargo supieron afrontar la situación y ejercer su rol de cuidadores primarios.

"No me podía adaptar, pero ya ahorita ya... ya no se me hace trabajo, pues antes me ponía nerviosa y me hice el ánimo y pues ya ahorita ya" E_2

Una vez que los cuidadores aceptaron la nueva situación, el cuidado de sus familiares se normalizó y el tiempo trascurrido fue pieza clave.

"Ya lo tomo como una rutina normal, ya no es como algo que jay [...] Ya me acostumbré porque ya tenemos mucho tiempo" $E_{3} 3$

La adecuada utilización de las estrategias de afrontamiento incide sobre el bienestar del cuidador. A medida que los cuidadores emplearon las estrategias de afrontamiento a su favor, pudieron ir experimentando la adaptación y normalización de la nueva situación, a tal grado de sentirse adaptadas al cambio.

Por otra parte, en el proceso de afrontamiento que vivieron los cuidadores al inicio de su trayectoria de cuidado, fue fundamental la influencia de sus creencias religiosas. Por esto, las creencias y su valor son parte de las estrategias de afrontamiento que determinan la conducta humana dentro del proceso salud-enfermedad, y se toman como hilo conductor para establecer el nexo existente entre la psicología, la salud y la religión ${ }^{11}$.

"Yo siempre he dicho que Dios me da fortaleza y que me de valor y las fuerzas para seguir" E_3

"Tenemos confianza en Dios, si no fuera así quien sabe que haría él o yo, quizá me desesperaría" E_I "Dios nos ha dado la mano, donde quiera que vamos yo siempre estoy conectado a él nunca me ha dejado ni yo a él, porque si te sueltas olvídate, te va mal, te tienes que agarrar bien, te tienes que aferrar a él, porque es lo único que tienes dentro de ti, la fuerza de Dios" E_7

Como puede verse en los discursos de los cuidadores se evidencia que experimentaron un afrontamiento activo ante la situación de su familiar, porque asumieron su rol con valor y responsabilidad, además emplearon sus recursos, el tiempo y su creencia en un ser superior para encontrar fortaleza y manejar la situación.

\section{Categoría 2.- Etapa de Adaptación}

Una vez vivido el inicio de la trayectoria del cuidado, en donde los familiares se convirtieron en cuidadores primarios, se presentó el fenómeno de la adaptación, el cual es el proceso de acomodarse y ajustarse a diversas circunstancias 0 condiciones ${ }^{12}$. Es decir, los cuidadores realizaron ajustes que consideraron necesarios como parte de su proceso de adaptación.

De acuerdo con Fhon ${ }^{13}$ los cuidadores reconocen que a medida que se extiende el tiempo con la tarea de cuidado, comienzan a experimentarse cambios en distintas áreas de la vida, como dejar de hacer sus actividades de la vida diaria por atender o acompañar a su familiar. Los ajustes que hicieron estos cuidadores al brindar cuidado, se vieron reflejados en su vida personal y la dinámica familiar.

"...dejé a mis amigas, dejé el ejercicio, dejé de ir a caminar, dejé de ir hasta con mi familia porque no podía yo dejarlo solo en ese instante, este, y porque, porque tenía que estar aquí.... Uno tiene que dejar todo" $E 3$

"No más dejé de trabajar" E_6

Los ajustes en la dinámica familiar estuvieron relacionados con el cuidado proporcionado a los familiares, por lo que no solo llevaron a cabo cambios en su vida personal y social, sino además aprendieron a emplear estrategias relacionadas con lo que les preocupaba del cuidado de su familiar; atendieron las necesidades de alimentación, medicación y el aseo personal, principalmente.

Respecto a la alimentación, el cuidado que proporcionaron estuvo orientado a lograr que su familiar consumiera lo adecuado sin riesgos, dichas estrategias fueron evitar la compra de los alimentos prohibidos para su familiar. Además consumían de la misma comida que su familiar para apoyarlo con la decisión de adaptarse al tratamiento: 
"Esa es mi estrategia, no comprar esto y ya sé que no habiendo aquí en la casa no va a comerlo, mejor no compro las cosas que le hace daño y así no come" E_3

"No nos gustan las verduras, pero tenemos que consumirlas por él" $E_{-} 1$

Las narraciones dan cuenta no solo del aspecto técnico del cuidado, es decir los cambios que tuvieron que hacer en el consumo y la forma de preparación de los alimentos, sino que además demostraron su apoyo hacia la persona que cuidan, ya que ellas mismas cambiaron sus hábitos alimenticios para que sus familiares lograran adaptarse a su dieta.

Asimismo, los cuidadores consideraron que sus familiares deben recibir cuidados relacionados con la restricción de alimentos y líquidos. Debido al cambio repentino de la alimentación, los pacientes con ERC buscan la manera de seguir ingiriendo todos aquellos alimentos que les fueron restringidos. Por tanto, las cuidadoras estuvieron pendientes de ellos procurando hacerles una llamada de atención en momentos en los que éstos querían consumir algo desaconsejado:

"A veces que quiere comer algo que le hace daño, le digo y se enoja. Mejor no le digo nada" E_4

"Tengo que cuidarlo, tengo que estar al pendiente de él, qué toma, qué come o qué no come y así" E_6

Por otro lado, los cuidadores dieron especial importancia a la higiene corporal de sus familiares, lo que se traduce en cuidados de su catéter venoso central. Los cuidadores permanecieron con la búsqueda de estrategias para brindar el mejor cuidado posible para sus familiares. Dentro de dichas estrategias buscaron que sus familiares se asearan diariamente sin mojar el sitio de inserción del catéter.

"No más le ponía un parchecito que se le atoraba cuando tenía su catéter. Después me dijo una señora ponle una toalla femenina y él me decía que es eso, me respingaba, pero no se le mojaba" $E \_5$

"Lo limpiaba con trapos mojados, porque me dijeron que para que no le fuera a entrar agua" $E \_6$

Las cuidadoras emplearon diferentes estrategias de cuidado al momento del aseo del catéter. Algunas de ellas denotan riesgos de infección para el catéter, por lo que es importante que los profesionales de enfermería lleven a cabo un adecuado asesoramiento a los cuidadores respecto al manejo que deben de tener con relación al catéter.

En relación a la medicación, las cuidadoras se aseguraban de que el familiar ingiriera sus medicamentos pres- critos, supervisaban que los tomara en la hora adecuada y que fuera el medicamento correcto:

"...el miércoles tiene que desayunar antes de que le den la hemodiálisis y tomar sus pastillas normales..." E 3

"Regresa de la hemodiálisis, come bien, le doy sus pastillas, y ya se acuesta y se duerme" $E \_2$

Por otro lado, la interacción que tuvieron los cuidadores con su familiar les permitió identificar otro tipo de necesidades, las cuales estaban relacionadas con la vida social del enfermo. Al reconocer esta situación los cuidadores se transformaban en acompañantes de sus familiares, debido a que los cuidadores consideraban que eso les proporcionaba satisfacción y que era bueno para ellos:

"Pues yo nada mas de acompañante, de que voy a acompañarlo a donde va, ya vamos al hospital ya vamos a hemodiálisis, pues a donde va" $E_{-} 4$

"Yo me sentía bien o sea normal digamos, yo siempre la acompañaba... No necesitaba mucho de los cuidados, nomás la compañía" E_3

Partiendo de sus comentarios, consideramos que para ellos el acompañamiento a su familiar fue otra manera de cuidar.

Por otra parte, en relación con los ajustes realizados en la dinámica familiar se vio implícito el cuidado que brindaron los cuidadores, ya que de acuerdo con Ramos del $\mathrm{Rio}^{14}$ en dicho cuidado se requiere que los cuidadores realicen múltiples intervenciones; éstas incluyen la satisfacción de necesidades de cuidado de la salud, psicosociales y económicas. Las de salud están relacionadas con el manejo de signos y síntomas de la enfermedad, con regímenes prescritos para problemas reiterativos, concomitantes e irreversibles como el dolor y la invalidez; las psicosociales incluyen las reacciones al afrontamiento de la enfermedad, la prevención o minimización del aislamiento; y las económicas que son la tenencia y consecución de recursos que siempre se están incrementando de forma constante e impredecible ${ }^{15}$. Por lo que este cuidado demandó que los cuidadores realizaran múltiples actividades que requirieron de su esfuerzo, y que en determinado momento, les ocasionaron problemas de salud por presión psicológica (sensaciones de malestar, de sobrecarga, depresión, cansancio).

"Me sentía agotada. En el cuarto se acostaba y escuchaba algo y me tenía que levantar rápido para ver cómo estaba" $E \_6$

"Primero sí me sentía cansada porque sentía que todo me dolía, era como el estrés" E_3 
Las decisiones que tomaron los cuidadores y los ajustes que realizaron para adaptarse a su nueva forma de vida, les llevaron a experimentar sentimientos; éstos son el resultado de la interacción con la emoción. El sentimiento implica una participación de la conciencia y por tanto del cerebro más evolucionado. Algunos dicen que es el componente cognitivo de la emoción ${ }^{16}$.

Los cuidadores manifestaron haber sentido preocupación y temor por su familiar, derivado de los pensamientos de que sus familiares pudiesen pasarla mal cuando ellos no estaban a su lado.

"Porque tengo pendiente de él, me voy y sé que está malo" E 2

"Siento que le va a pasar algo si lo dejo solo" E_6

No obstante que los cuidadores expresaron sentir cansancio en algún momento del cuidado, se considera que fue difícil para ellos admitir esa condición física de su cuerpo. Por lo que su cansancio fue justificado y/o minimizado por los cuidadores por dos motivos para el cuidado: el primero relacionado con el amor y el segundo con el rol que juegan en la vida de su familiar. Esta situación ha sido descrita por Ibarrola ${ }^{16}$, ya que considera que las razones por las que se brinda ayuda a un familiar, se dice que por "amor incondicional" es la razón principal. Sin embargo, es posible que la primera respuesta sea "porque es su obligación". Asimismo, las personas que cuidan a un familiar están de acuerdo en que se trata de un deber moral que no debe ser eludido y que existe una responsabilidad social y familiar.

En ese sentido, los cuidadores expresaron que los motivos por los que brindaron el cuidado a sus familiares fue por amor, por el dolor que representaba ver el sufrimiento de un ser amado, asimismo porque para ellos, el cuidado, es su labor u obligación como esposas o madres.

"Es una obligación porque es mi hijo y me duele, es mi sangre y cómo lo voy a dejar que se me vaya"E_6

"Pues quien más lo va a cuidar más que yo que soy su compañera" E_2

El amor y el rol fueron determinantes en el cuidado de sus familiares, debido a que esas condiciones les permitieron a los cuidadores transformar el cansancio en fuerza para no decaer en el cuidado.

Otro aspecto importante en este momento de la trayectoria del cuidado fue que los cuidadores manifestaron que poseían cierta regulación emocional y autocontrol que les permitió dirigir adecuadamente sus emociones y eso las fortaleció para sobresalir de la situación. Esta competencia le proporciona al cuidador la serenidad y la lucidez necesarias para afrontar situaciones estresantes y le ayuda a mantenerse imperturbable ante situaciones críticas ${ }^{15-16}$.

"Como dé lugar, de una forma u otra, lo tengo que sacar adelante porque es mi hijo y me duele, voy a seguir haciendo todo por él" E_6

En la etapa intermedia de su trayectoria del cuidado los cuidadores vivieron la adaptación a la nueva forma de vida con ajustes en sus actividades diarias en torno al cuidado de sus familiares, tuvieron sus sentimientos que de alguna manera, junto con sus motivos para cuidar, les resultó posible manejar la situación para continuar con el cuidado. Asimismo en este momento de su trayectoria del cuidado, los cuidadores dieron un significado al acto de cuidar que estuvieron brindando, lo cual les permitió experimentar satisfacción de poder ayudar a su familiar. Además manifestaron que lo hacían con agrado para ellos y sintieron placer de ser parte del cuidado y recuperación percibida en esta etapa:

"Estoy satisfecha porque pues no se me ha complicado mucho, para mí ha sido placentero, yo me siento a gusto, lo hago con gusto" $E \_3$

"Para mí el cuidarlo es una satisfacción, el poder ayudarlo y que yo también me puedo mover (se observan lágrimas en los ojos)" $E_{-} 7$

Las cuidadoras experimentaron sentirse bien y contentas con solo ver a su familiar con bienestar y en condiciones de salud estables:

"Me siento contenta porque él cada día está mejor" E 3

"Ahorita, gracias a Dios, ya me siento bien porque lo veo bien también a él y mientras que él esté bien yo creo que también yo voy a estar" E_4

Los ajustes realizados ante la adaptación para brindar el cuidado y la percepción de estabilidad en la salud de su familiar gracias a dichos cuidados, es lo que vivieron los cuidadores en la etapa dos de la trayectoria del cuidado. Posteriormente experimentaron la complejidad de la etapa del desenlace, en donde hubo presencia de diversos duelos.

\section{Categoría 3.- La etapa final/desenlace: del cuidado anticipado al duelo patológico}

El primer duelo vivido fue el anticipado; los cuidadores experimentaron sensaciones y pensamientos relacionados con la idea de la muerte de su familiar. 


\section{"Yo sí sé que se va a morir y como tal tengo que aceptarlo que algún día va a morir" E_10}

Los cuidadores tuvieron sensaciones y pensamientos relacionados con el momento inevitable de la pérdida de su familiar. Al considerar ellos que la insuficiencia renal es una enfermedad crónica y con la connotación de "terminal", atribuyeron este término a la muerte que podría sufrir su familiar en cualquier momento.

"Desde el principio tenía en mente de que iba a suceder en algún momento sin embargo estábamos con la duda de cuando en que momento existiría algunas señales" E_9

"Estábamos conscientes de que algún momento podía llegar la muerte" $E_{-} 12$

El duelo anticipado generalmente es vivido por los familiares del enfermo, sobre todo en el caso de enfermedades terminales y/o crónicas degenerativas, ya que les ayuda a tomar conciencia de cuanto está sucediendo, a liberar los propios estados de ánimo y a programar el tiempo en vista de la inevitable muerte ${ }^{17}$. Este duelo puede manifestarse a través de diferentes formas como tristeza, ansiedad, intentos de reconciliarse y deseos de reconstituir o fortalecer los lazos familiares, por lo que también ofrece a las personas implicadas la oportunidad de compartir sentimientos y prepararse para la despedida ${ }^{18}$.

"...desde entonces yo traía un pensamiento de que podía tratarlo de una manera diferente, hacerle cambiar su mente porque desde que el médico le determino, pensó en morirse y empezó a decaer su autoestima... todo empezó a decaer..." E_8

No obstante que se puede pensar que todo proceso de duelo conlleva al sufrimiento, algunos autores mencionan que el duelo anticipado es beneficioso, debido a que es una respuesta adaptativa positiva porque facilita a las personas la posibilidad de "ensayar" el papel del duelo real y comenzar a trabajar con los profundos cambios que acompañan a la pérdida cuando ocurre, mitigando así el trauma asociado al duelo postmortem ${ }^{19}$.

Por otra parte, hubo cuidadores que experimentaron un duelo real, al recibir la noticia de la muerte de su familiar, presentándose con esto la última etapa de la trayectoria del cuidado.

"Tu hermano ya falleció (triste) es como que me cayó un balde de agua helada, no puede ser ¿cómo?, ¿por qué?" $E_{-} 8$.

"Tienen que ser fuertes que más vamos hacer, nada se puede hacer ya" E_9
El duelo es un proceso normal que se da por una pérdida significativa, se inicia desde el momento en que se da la pérdida, se soluciona cuando se acepta el pasado sin quererlo cambiar; se vive el presente y se planea el futuro sin aquello que se ha perdido ${ }^{20}$.

"Después de que ya no está ahora a quién aplicaré todo eso, ahora con quién voy a platicar en confianza, a quién le diré hermano te quiero pero pues lejos de perder yo siento que gane demasiado" E_8

Los momentos que rodean la muerte de un ser querido, son de mucha intensidad emocional y tienen gran impacto en los familiares, por lo que los cuidadores vivieron momentos de dificultad y buscaron ayuda profesional.

"Sentía yo como ganas de que una persona platicara conmigo, para ya no seguir pensando" $E 9$

"Yo tomé la decisión de ir al psicólogo, necesité ayuda, pues si es muy necesario" $E \_8$

Los cuidadores consideraron como una necesidad relevante el apoyo psicológico, ya que generalmente dentro de los servicios y/o centros especializados, después del fallecimiento del paciente con tratamiento de hemodiálisis, los cuidadores quedan en el olvido, y eso a su vez repercute en su salud.

A manera de conclusión se puede considerar que el rol de la mujer como cuidadora de sus familiares enfermos permanece en el tiempo. Son las mujeres quienes en su mayoría viven el impacto del cuidado del otro. En ese proceso de convertirse en cuidadoras experimentaron el proceso de afrontamiento junto con diversidad de sentimientos como temor, preocupación e incertidumbre por un lado y por otro, la satisfacción de ayudar. Los cuidadores tuvieron recursos que emplearon a su favor para afrontar la nueva situación y adaptarse: dichos recursos fueron el aceptar la situación y asumir su rol de cuidadoras, el tiempo y la creencia en un ser superior en quien encontraron fortaleza para salir adelante. Asimismo las cuidadoras desarrollaron habilidades y estrategias de cuidado de su familiar sobre la alimentación, medicación y el aseo personal. Además las cuidadoras identificaron otra necesidad de cuidado requerido por sus familiares, la compañía a sus tratamientos o salidas recreativas, por tanto, dieron entonces otro significado al acto de cuidar; para las cuidadoras acompañar es sinónimo de cuidado.

El conocer la trayectoria de cuidado que viven los cuidadores primarios permite identificar las necesidades tanto de conocimiento como de apoyos que demandan, para afrontar la función que realizan en cada una de 
las etapas por las que atraviesa la descrita trayectoria. Asimismo, faculta vislumbrar las posibles consecuencias que tendrán dichos cuidadores en sus diferentes dimensiones como seres humanos, lo que da la oportunidad de prever mediante un acompañamiento adaptado a sus necesidades, y de esa manera contribuir a disminuir las repercusiones del cuidado que influyen en la calidad de vida del cuidador, especialmente la sobrecarga que llegan a experimentar muchos de ellos.

Recibido: 18-03-19

Revisado: 15-04-19

Modificado: 8-05-19

Aceptado: 25-05-19

\section{Bibliografía}

1. Instituto Nacional de Estadística y Geografía. Características de las defunciones registradas en México durante 2017. [Consultado 15 enero 2019]. Disponible en: https://www.inegi.org.mx/contenidos/saladeprensa/boletines/2018/EstSociodemo/ DEFUNCIONES2017.pdf.

2. Álvarez Villareal M, Chocarro González L, Velarde García JF, Palacios Ceña D. La experiencia de ser portador de un catéter venoso central para hemodiálisis: Estudio cualitativo. Enferm Nefrol. 2018;21(2):146-154. [Consultado 25 enero 2019]. Disponible en: http://scielo.isciii.es/scielo.php?scrip$\mathrm{t}=$ sci_abstract\&pid=S2254-28842018000200006.

3. Huérfano Martínez $D$, Álvarez Zambrano $D$, Arias Silva E, Carreño Moreno S. Necesidades de personas en terapia de diálisis y sus cuidadores: Revisión integrativa. Enferm Nefrol. 2018;21(2):155-165. [Consultado 25 enero 2019]. Disponible en: http:// scielo.isciii.es/scielo.php?script=sci_arttext\&pi$\mathrm{d}=$ S2254-28842018000200007.

4. Sánchez Herrera B, Carrillo González GM, Barrera Ortiz L. Carga del cuidado de la enfermedad crónica no transmisible. Rev Aquichán. 2013; 13(2):247260. [Consultado 17 noviembre 2018]. Disponible en: http://www.redalyc.org/pdf/741/74128688001. pdf.

5. Velázquez Pérez Y, Espín Andrade AM. Repercusión psicosocial y carga en el cuidador informal de personas con insuficiencia renal crónica terminal. Rev Cubana de Salud Pública. 2014; 1(40):3-17. [Consultado 17 noviembre 2018]. Disponible en: http://scielo.sld.cu/scielo.php?script=sci_arttext\&pid=S0864-34662014000100002.

6. Asociación Médica Mundial. Declaración de Helsinki; Principios éticos para la investigación médica en seres humanos. Adoptado por la 64a Asamblea Médica Mundial. Fortaleza, Brasil. 2013. [Consultado 15 noviembre 2018]. Disponible en: http:// www.isciii.es/ISCIII/es/contenidos/fd-investigacion/ $\mathrm{fd}$-evaluacion/fd-evaluacion-etica-investigacion/Declaracion-Helsinki-2013-Esp.pdf.

7. Secretaria de Salud, Ley General de Salud. Título Quinto. México. [Consultado 15 noviembre 2018]. Disponible en: http://www.salud.gob.mx/cnts/pdfs/ LEY_GENERAL_DE_SALUD.pdf.

8. Noreña $A L$, Alcaraz-Moreno $N$, Rojas JG, Rebolledo-Malpica D. Aplicabilidad de los criterios de rigor y éticos en la investigación cualitativa. Rev Aquichan. 2012;12(3):263-74. [Consultado 2 noviembre 2018]. Disponible en: http://www.redalyc.org/ articulo.oa?id=74124948006.

9. Pérez P, González A, Mieles I, Uribe AF. Relación del apoyo social, las estrategias de afrontamiento y los factores clínicos y sociodemográficos en pacientes oncológicos. Pensamiento Psicológico. 2017;15(2):41-54. [Consultado 15 noviembre 2018]. Disponible en: https://dx.doi.org/10.11144/ Javerianacali.PPSI15-2.rase.

10. Amarís Macías M, Madariaga Orozco C, Valle Amarís M, Zambrano J. Estrategias de afrontamiento individual y familiar frente a situaciones de estrés psicológico. Psicología desde el Caribe. 2013;30(1):123-145. [Consultado 15 noviembre 2018]. Disponible en: https://www.redalyc.org/articulo.oa? id=21328600007.

11. Flores-Carvajal D, Alfonso Urzúa M. Propuesta de evaluación de estrategias de afrontamiento ante la enfermedad crónica en adolescentes. Rev Chil Pediatr. 2015;87(3):169-174. [Consultado 25 enero 2019]. Disponible en: http://dx.doi.org/10.1016/j. rchipe.2015.08.007. 
12. Real Academia Española. Diccionario de la lengua española. [Consultado 25 enero 2019]. Disponible en: https://dle.rae.es/?id=0hMBUwM.

13. Fhon J, Gonzales Janampa J, Mas Huaman $T$, Marques S, Rodrigues R. Sobrecarga y calidad de vida del cuidador principal del adulto mayor. Avances en Enfermería 2016;34(3):251-8. [Consultado 25 enero 2019]. Disponible en: https://doi. org/10.15446/av.enferm.v34n3.58704.

14. Ramos del Río B, Jarillo Soto EC. El cuidado informal de personas con enfermedad renal crónica. Una mirada desde la salud colectiva y la teoría de las representaciones sociales. En-claves del pensamiento. 2014;8(15):195-216. [Consultado 2 febrero 2019]. Disponible en: http://www. scielo.org.mx/scielo.php?script=sci_arttext\&pi$d=S 1870-879 \times 2014000100195 \& \operatorname{lng}=e s \& t \operatorname{lng}=e s$.

15. Achury DM, Castaño Riaño HM, Gómez Rubiaño LA, Guevara Rodríguez NM. Calidad de vida de los cuidadores de pacientes con enfermedades crónicas con parcial dependencia. Rev. Investigación en Enfermería: Imagen y Desarrollo. 2011; 1(13):27-46. [Consultado 25 enero 2019]. Disponible en: http:// www.redalyc.org/pdf/1452/145221282007.pdf.

16. Ibarrola B. Competencias emocionales de los cuidadores de personas mayores. IMSERC0. 2014. Disponible en: http://envejecimiento.csic.es/documentos/documentos/ponencias-BegonaIbarrola-Competenciasemocionales-cuidadores-personasmayores-06-2014.pdf.
17. Martínez Sarantez A, Turniell Castillo D, Gutiérrez Mendoza S, Solís Zúniga F. (2018). Experiencias de duelo anticipado en familiares de pacientes con enfermedad oncológica en etapa terminal. Revista Científica de FAREM-Estelí. 2018;(26):77-83. [Consultado 2 febrero 2019]. Disponible en: https:// doi.org/10.5377/farem.v0i26.6444.

18. Morer Bambaa B, Alonso Gómez R, Oblanca Beltrán M. El duelo y la pérdida en la familia. Revisión desde una perspectiva relacional. Rev Psicoterapia relacional e intervenciones sociales. 2017;36:1123. [Consultado 22 febrero 2019]. Disponible en: http://www.redesdigital.com.mx/index.php/redes/ article/view/196/159.

19. Asociación Mexicana de Tanatología. Curar el dolor de la muerte y el de la desesperanza. Disponible en: http://www.tanatologia-amtac.com/.

20. Ornelas Tavares PE. Estrategias de afrontamiento y resiliencia en cuidadores primarios con duelo. Psicología y salud. 2016;26(2):177-184. [Consultado 22 febrero 2019]. Disponible en: http://psicologiaysalud.uv.mx/index.php/psicysalud/article/ view/2193/3921.

Este artículo se distribuye bajo una Licencia Creative Commons Atribución-NoComercial 4.0 Internacional. https://creativecommons.org/licenses/by-nc/4.0/ 